\title{
Duality, area-considerations, and the Kalai-Smorodinsky solution
}

\author{
Emin Karagözoğlu ${ }^{\mathrm{a}, \mathrm{b}, *}$, Shiran Rachmilevitch ${ }^{\mathrm{c}}$ \\ ${ }^{a}$ Bilkent University, Department of Economics, 06800 Çankaya, Ankara, Turkey \\ ${ }^{\mathrm{b}}$ CESifo, Poschingerstr. 5, 81679 Munich, Germany \\ ${ }^{\mathrm{c}}$ Department of Economics, University of Haifa, Mount Carmel, 31905 Haifa, Israel
}

\section{A R T I C L E I N F O}

\section{Article history:}

Received 19 September 2016

Received in revised form

6 November 2016

Accepted 6 November 2016

Available online 17 November 2016

\section{Keywords:}

Axioms

Bargaining problem

Dual bargaining problem

Egalitarianism

Equal-area solution

Kalai-Smorodinsky solution

\begin{abstract}
A B S T R A C T
We introduce a new solution concept for 2-person bargaining problems, which can be considered as the dual of the Equal-Area solution (EA) (see Anbarc1 and Bigelow (1994)). Hence, we call it the Dual EqualArea solution (DEA). We show that the point selected by the Kalai-Smorodinsky solution (see Kalai and Smorodinsky (1975)) lies in between those that are selected by EA and DEA. We formulate an axiom area-based fairness - and offer three characterizations of the Kalai-Smorodinsky solution in which this axiom plays a central role.
\end{abstract}

(c) 2016 Elsevier B.V. All rights reserved.

\section{Introduction}

In a bargaining problem two players need to agree on a utility allocation from a feasible set of allocations, $S \subset \mathbb{R}_{+}^{2}$. Failure to reach an agreement leads to a status quo utility of zero for each player. A bargaining solution describes how the players solve every conceivable bargaining problem; formally, a solution is a function that chooses a unique point from every such $S$. One basic requirement on a bargaining solution is fairness, in the sense that whenever more options become feasible, no one should get hurt. Since a bargaining problem is an infinite object, there are many ways of defining how "more options become feasible". [4] proposed the following fairness requirement, which is based on the area of $S$ : if the area of $S$ increases, no one should get hurt. Moreover, they showed that there is a unique solution (equal-area solution) which satisfies this property (area monotonicity) and strong Pareto optimality on the domain of convex problems. The equal-area solution assigns to each $S$ the point on its frontier, $x$, such that the line segment between the origin and $x$ splits $S$ into two parts of equal areas. Informally, the equal-area solution can be considered as an application of the egalitarian principle on an area measure of concessions.

\footnotetext{
* Corresponding author at: Bilkent University, Department of Economics, 06800 Çankaya, Ankara, Turkey.

E-mail address: karagozoglu@bilkent.edu.tr (E. Karagözoğlu).
}

[2,5], and [3] provided noncooperative and dynamic foundations for the equal-area solution.

We introduce a new concept into the bargaining literature duality - and apply it to the equal-area solution. The resulting solution is the dual equal-area solution. This solution applies the egalitarian principle on an area measure of aspirations. We formulate a requirement - area-based fairness - which stipulates that when these two solutions propose the same point, then this point should be chosen as the solution point. We derive three characterizations of the Kalai-Smorodinsky solution (see [9]) on the basis of area-based fairness and some standard axioms.

Section 2 describes the bargaining model. Section 3 introduces duality. Section 4 introduces area-based fairness. The characterization results are in Section 5. Finally, Section 6 concludes.

\section{The bargaining model: definitions}

The following is a simple version of the bargaining model in [10]. A bargaining problem is a compact and comprehensive set $S \subset \mathbb{R}_{+}^{2}$ that contains $\mathbf{0} \equiv(0,0)$ as well as some $x$ with $x>\mathbf{0}$, where vector inequalities are defined as follows: $u R v$ if and only if $u_{i} R v_{i}$ for each $i$, for both $R \in\{>, \geq\}$, and $u \supsetneqq v$ if and only if $u \geq v$ and $u \neq v$. The set $S$ (the feasible set) is a menu of utility-pairs out of which a single point needs to be agreed upon. Agreement on $x$ means that the bargaining problem is resolved and each agent $i$ obtains the utility $x_{i}$; failure to reach an agreement leads to the status quo utilities, $\mathbf{0}$. The assumption $S \cap \mathbb{R}_{++}^{2} \neq \varnothing$ implies that 


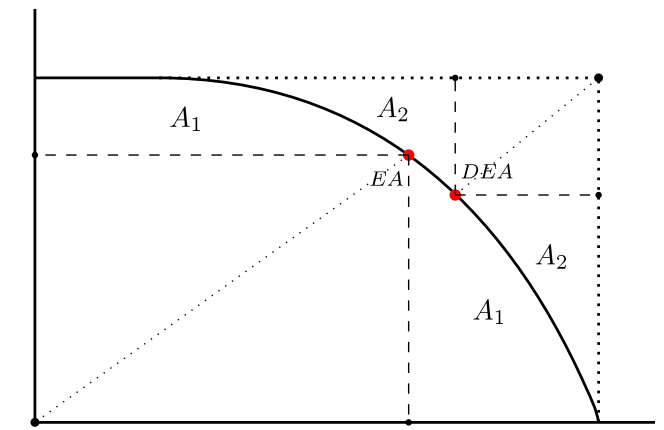

$(0,0)$

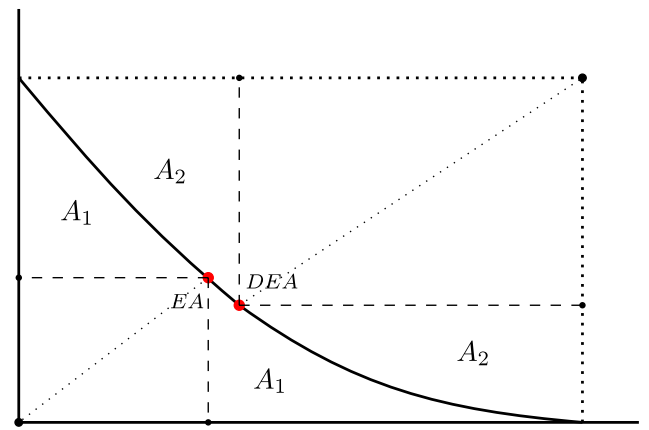

$(0,0)$

Fig. 1. EA and DEA in convex and non-convex problems.

the status quo can be abandoned in a way that makes both agents better off (relative to the status quo). The assumption that $S$ is comprehensive - namely, that $x \in S \Rightarrow y \in S$ for every $y$ that satisfies $\mathbf{0} \leq y \leq x$ - means that utilities can be freely disposed (down to the status quo levels). Given a problem $S$, the ideal point of $S, a(S)$, is defined by $a_{i}(S) \equiv \max \left\{s_{i}: s \in S\right\}$. The number $a_{i}(S)$ is called agent $i$ 's ideal payoff. The collection of problems is denoted by $\mathcal{B}$. Let $\mathscr{B}^{c o} \equiv\{S \in \mathscr{B}: S$ is convex $\}$.

A solution on a bargaining domain $\mathscr{D} \subset \mathscr{B}$ is a function $f: \mathscr{D} \rightarrow$ $\mathbb{R}_{+}^{2}$ that satisfies $f(S) \in S$ for every $S \in \mathscr{D}$.

Given a function $\psi: \mathbb{R}^{2} \rightarrow \mathbb{R}^{2}$ and a set $C \subset \mathbb{R}^{2}$, we let $\psi \circ C \equiv\{\psi \circ c: c \in C\}$. A solution $f$ is scale covariant if for every problem $S$ and every pair of positive linear transformations, $l=\left(l_{1}, l_{2}\right)$, it is true that $f(l \circ S)=\operatorname{lof}(S)$. A solution $f$ satisfies contraction independence if $f(T) \in S \subset T \Rightarrow f(S)=f(T)$. Contraction independence has first been introduced by [10] (under the name independence of irrelevant alternatives). Restricted contraction independence, introduced by [12], narrows the scope of the axiom: it imposes the requirement of contraction independence only on pairs of problems, $\{S, T\}$, that in addition to the condition of contraction independence share the same ideal point. Another axiom that we will use and that also imposes a restriction on nested problems with a common ideal point is restricted monotonicity (introduced by [13]). It requires $[S \subset T \& a(S)=a(T)] \Rightarrow f(S) \leq f(T)$. Finally, $f$ is continuous if for each sequence of problems $\left\{S_{k}\right\}$ that converges to a problem $S$ in the Hausdorff topology, $f\left(S_{k}\right)$ converges to $f(S)$.

Given $v>\mathbf{0}$, the comprehensive hull of $\{v\}$ is $\operatorname{comp}\{v\} \equiv\{x \in$ $\left.\mathbb{R}_{+}^{2}: x \leq v\right\}$. Given a set $A \subset \mathbb{R}_{+}^{2}, \bar{A}$ denotes the closure of $A$.

\section{Duality}

Given a problem $S$ with $S \neq \operatorname{comp}\{a(S)\}$, we define the dual problem of $S$ as $D(S) \equiv \overline{\operatorname{comp}\{a(S)\} \backslash S \text {. }}$

At an informal level, one can think of the following story as underlining a bargaining problem: the agents "start" at the origin, and they need to move forward, towards the frontier, and reach an agreed-upon point. Then, the informal story corresponding to the dual problem is that the agents "start" at the ideal point and they need to concede to move towards the frontier and reach a feasible point.

We define the duality transformation, $\phi_{S}=\left(\phi_{S, 1}, \phi_{S, 2}\right)$, by $\phi_{S, i}(x) \equiv-\left(x-a_{i}(S)\right)$. The transformations $\phi_{S, 1}$ and $\phi_{S, 2}$ shift the ideal point to the origin and then "mirror" the resulting set, carrying it from the south-west quadrant to the north-east one. Note that for every $S \in \mathscr{B}$ such that $S \neq \operatorname{comp}\{a(S)\}$ we have $\phi_{S} \circ D(S) \in$ $\mathscr{B}$. More generally, a bargaining domain $\mathscr{D}$ is closed under duality if $[S \in \mathbb{D}] \&[S \neq \operatorname{comp}\{a(S)\}] \Rightarrow \phi_{S} \circ D(S) \in \mathscr{D}$. In this paper, we consider two domains: the grand domain $\mathscr{B}$ and its subset $\mathscr{B}^{\text {co }}$. The former is closed under duality, whereas the latter is not.
Let $\mathscr{D}$ be a domain such that (i) it is closed under duality and (ii) no $S \in \mathscr{D}$ contains a boundary with a segment parallel to an axis. Let $f$ and $\tilde{f}$ be two solutions on $\mathscr{D}$. The solution $\tilde{f}$ is the dual of $f$ on $\mathscr{D}$ if the following holds for all $S \in \mathscr{D}$ :

$\tilde{f}(S)=\phi_{S}^{-1} \circ f\left(\phi_{S} \circ D(S)\right)$,

where $\phi_{S, i}^{-1}$ is the inverse of $\phi_{S, i}$. In words, duality says that applying $\tilde{f}$ to $S$ is same as "standing at $a(S)$ " and applying $f$ to $D(S)$. Requirement (i) makes sure that $\phi_{S} \circ D(S)$ on the RHS of (1) belongs to $\mathscr{D}$. Requirement (ii) guarantees that 0 is the "ideal point" of $D(S)$.

The egalitarian solution (see [8]) and the equal-loss solution (see [6]) are two solutions that are duals of each other. The former assigns to each $S$ the intersection point of its (weak) Pareto frontier and the $45^{\circ}$-line, whereas the latter assigns to each $S$ the intersection point of its (weak) Pareto frontier and the $45^{\circ}$ line drawn from $a(S)$. On the other hand, the Kalai-Smorodinsky solution, which is defined by $K S(S) \equiv \max \{\lambda: \lambda a(S) \in S\} \times a(S)$ is dual to itself (i.e., Eq. (1) holds with $f=\tilde{f}=K S$ ) on the domain of problems with a boundary that does not contain a segment parallel to an axis.

\section{Area-based fairness}

The equal-area solution (see [4]) assigns to each $S$ the point of its Pareto frontier, $x$, such that the segment $\operatorname{conv}\{\mathbf{0}, x\}$ splits $S$ into two subsets of equal areas (see Fig. 1). In this paper, we introduce the dual of this solution, which we call the dual equal-area solution. For each $S$, this solution assigns the Pareto efficient $x$ such that $\operatorname{conv}\{x, a(S)\}$ splits $D(S)$ into two subsets of equal areas (see Fig. 1). We denote the equal-area solution and its dual by $E A$ and $D E A$, respectively.

As we mentioned in the Introduction, for convex $S, E A$ is the only strongly Pareto optimal solution satisfying the requirement that no one loses when the area of $S$ increases. Analogously, DEA satisfies the following property: when the area of $S$ decreases and the ideal point is unchanged, no one benefits. DEA satisfies some standard axioms such as Pareto optimality, symmetry, and scale covariance.

$E A$ and $D E A$ demonstrate that there are at least two ways to define egalitarianism on the basis of areas: the original way of [4] and the dual way of $D E A$ introduced here. We seek to merge them into a single fairness criterion. We believe that any reasonable merging would adhere to the following requirement:

Definition 1. A solution $f$ satisfies area-based fairness if for each $S$ the following holds:

$E A(S)=D E A(S)=x \Rightarrow f(S)=x$.

That is, in those cases where the two solutions agree, the areabased fairness property requires this agreed-upon point to be the solution-point. 


\section{Characterizations}

The following results use the fact that $D E A$ satisfies scale covariance (Lemma 1) and $K S_{i}(S)$ is sandwiched between $E A_{i}(S)$ and $D E A_{i}(S)$ for every $S \in \mathscr{B}$ and $i=1,2$ (Lemma 2). The statements and proofs of these lemmas are in the Appendix.

Theorem 1. A solution on $B$ satisfies area-based fairness and restricted contraction independence if and only if it is the KalaiSmorodinsky solution.

Proof. Clearly, KS satisfies both axioms (see Lemma 2 for areabased fairness). Conversely, let $f$ be a solution that satisfies them. Further, let $S \in \mathscr{B}$ and let $x=K S(S)$. We will prove that $f(S)=x$.

Let $V \equiv\left\{y \in \mathbb{R}_{+}^{2}: y \leq\left(a_{1}(S), x_{2}\right)\right\} \cup\left\{y \in \mathbb{R}_{+}^{2}\right.$ : $\left.y \leq\left(x_{1}, a_{2}(S)\right)\right\}$. By area-based fairness, $f(V)=x$. By restricted contraction independence, $f(S)=x$.

Under scale covariance, restricted contraction independence can be replaced by restricted monotonicity.

Theorem 2. A solution on $\mathscr{B}$ satisfies area-based fairness, restricted monotonicity, and scale covariance if and only if it is the Kalai-Smorodinsky solution.

Proof. Clearly, $K S$ satisfies all axioms. Conversely, let $f$ be a solution that satisfies them and let $S \in \mathcal{B}$. We will prove that $f(S)=K S(S)$. By scale covariance, we can assume that $a(S)=$ $(1,1)$. Let $T \equiv \pi \circ S$, where $\pi$ is the non-identity permutation; i.e., $\pi(a, b) \equiv(b, a)$. Let $V \equiv S \cap T$. Since $V$ is symmetric with respect to the $45^{\circ}$-line, area-based fairness implies that $f(V)=K S(V)=$ $K S(S)$. By restricted monotonicity, $f(S)=K S(S)$.

We do not know whether scale covariance can be dispensed with in Theorem 2. Nevertheless, Theorem 3 shows that, on the restricted domain of convex problems, it can be dispensed with.

Theorem 3. A solution on $\mathscr{B}^{c o}$ satisfies area-based fairness and restricted monotonicity if and only if it is the Kalai-Smorodinsky solution.

Proof. By Lemma 2, KS satisfies area-based fairness, and it clearly satisfies restricted monotonicity. Conversely, let $f$ satisfy both axioms; and let $S$ be a convex problem. We will prove that $f(S)=$ $K S(S)$. Let $V \equiv \operatorname{conv}\left\{\mathbf{0},\left(a_{1}(S), 0\right),\left(0, a_{2}(S)\right), K S(S)\right\}$. Note that $E A(V)=D E A(V)=K S(S)$. Therefore, by area-based fairness, $f(V)=K S(S)$ and by restricted monotonicity, $f(S)=K S(S)$.

\section{Closing comments}

The central solution in the bargaining literature is the Nash solution (see [10]), which assigns to each convex $S$ the maximizer of $x_{1} \cdot x_{2}$ over $x \in S$. The Kalai-Smorodinsky solution, in contrast to Nash solution, is well-defined also on the domain of nonconvex problems. There is a small literature that considers its characterization on this domain. We contribute to this literature, which includes [1,7,11], and [14].

Our paper also reveals the non-trivial fact that the KalaiSmorodinsky solution can be founded on the basis of area-related notions, a fact that can be viewed as a conceptual link between this solution and the Nash solution. An alternative area-related notion of duality between these solutions, one that has been recognized by [4], is as follows: the Nash solution selects the maximal point on the diagonal of the maximal-area rectangle that is contained in the bargaining problem, and the Kalai-Smorodinsky solution selects the maximal point on the diagonal of the minimal-area rectangle that contains the bargaining problem.

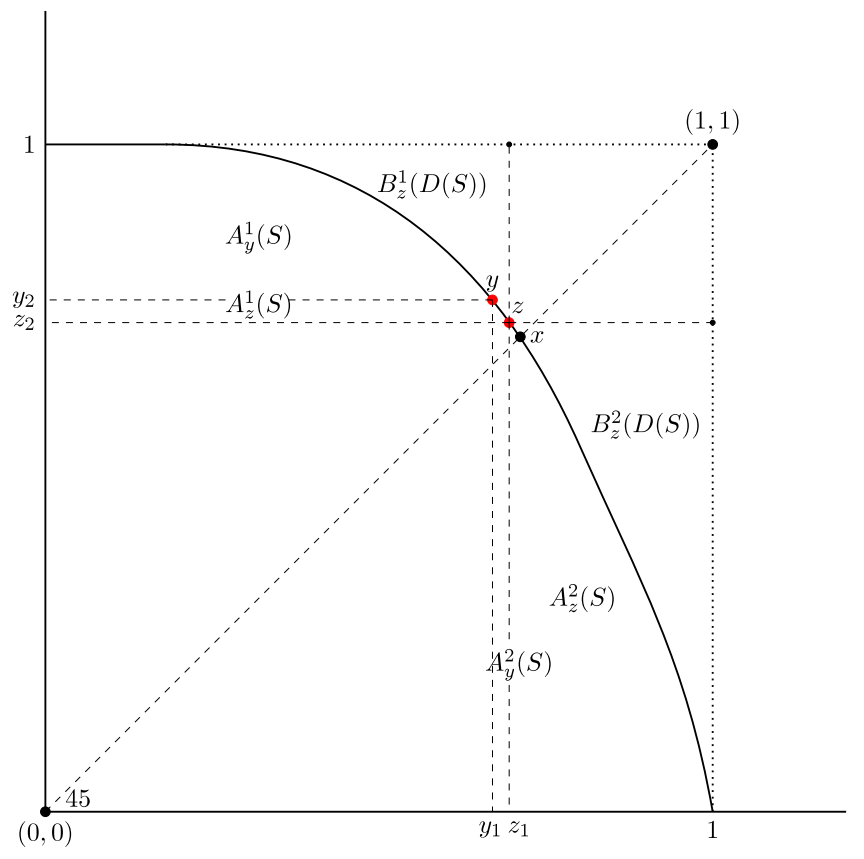

Fig. 2. Illustration of Case 4 in the Proof of Lemma 2.

\section{Acknowledgments}

The authors wish to thank William Thomson for detailed comments that had a significant impact on the research reported in this paper, Tarık Kara for discussions and insightful comments, Kerim Keskin for his help in the production of graphs, and an anonymous referee whose report was very helpful.

\section{Appendix}

Lemma 1. The dual equal-area solution satisfies scale covariance.

It is not hard to prove that DEA satisfies scale covariance and the proof follows the samearguments used to prove that $E A$ is scale covariant (see [4, pp. 137-138]). Hence, we omit the proof for the sake of brevity.

Lemma 2. The following holds for every $S \in \mathcal{B}$ :

$\min \left\{E A_{i}(S), D E A_{i}(S)\right\} \leq K S_{i}(S) \leq \max \left\{E A_{i}(S), D E A_{i}(S)\right\}$.

Proof. Let $S \in \mathscr{B}$. First, note that by scale covariance (satisfied by $K S, E A$, and $D E A)$, we can assume that $a(S)=(1,1)$. Now, let $x=K S(S), y=E A(S)$, and $z=D E A(S)$. Clearly, $x$ belongs to the $45-$ degree line in this normalized problem. We will prove that for each $i=1,2, \min \left\{y_{i}, z_{i}\right\} \leq x_{i} \leq \max \left\{y_{i}, z_{i}\right\}$. Without loss of generality, we focus on the case where $y$ is either on or above the 45-degree line.

Denote the equal areas generated by $y$ as $A_{y}^{1}(S)$ and $A_{y}^{2}(S)$. It is easy to see that $y$ also divides $D(S)$ into two (not necessarily equal) areas. Denote them by $B_{y}^{1}(D(S))$ and $B_{y}^{2}(D(S))$. Since $y$ is either on or above the 45-degree line, $A_{y}^{1}(S)+B_{y}^{1}(D(S)) \leq A_{y}^{2}(S)+B_{y}^{2}(D(S))$. But since $A_{y}^{1}(S)=A_{y}^{2}(S)$, this further implies $B_{y}^{1}(D(S)) \leq B_{y}^{2}(D(S))$. This inequality implies four possibilities concerning the location of $z$ on the frontier of $S$. Before analyzing these four cases, first note that if $y$ is on the 45-degree line (i.e., $y=x$ ), then because all weak inequalities above are satisfied as equalities, it follows that $z=x$ : so, we are done. Therefore, below we consider situations where $y$ is above the 45 -degree line. 
Case 1: $z=y$. The fact that $y$ is above the 45-degree line implies $B_{y}^{1}(D(S))<B_{y}^{2}(D(S))$. On the other hand, by definition, $B_{z}^{1}(D(S))=B_{z}^{2}(D(S))$. Hence, $z=y$ implies a contradiction.

By the same argument, the inequalities $y_{1}>z_{1}$ and $y_{2}<z_{2}$ (i.e., $z$ is to the north-west of $y$ ) also lead to a contradiction.

Case 2: $z$ is below the 45-degree line. Then, $x$ is sandwiched by $y$ and $z$.

Case 3: $x=z$. Note that $z$ divides $D(S)$ into two equal areas, denoted by $B_{z}^{1}(D(S))$ and $B_{z}^{2}(D(S))$. It also divides $S$ into two (not necessarily equal) areas. Denote them by $A_{z}^{1}(S)$ and $A_{z}^{2}(S)$. If $z$ is on the 45-degree line, then $A_{z}^{1}(S)+B_{z}^{1}(D(S))=A_{z}^{2}(S)+B_{z}^{2}(D(S))$. This implies that $A_{z}^{1}(S)=A_{z}^{2}(S)$, contradicting $y \neq z$. It is worthwhile emphasizing here that if any two of $\{x, y, z\}$ coincide, then they all coincide.

Case 4: $y_{1}<z_{1}$ and $y_{2}>z_{2}$ (i.e., $z$ is to the east of $y$ ) but $z$ is still above the 45-degree line. Since $z$ is above the 45-degree line, $A_{z}^{1}(S)+B_{z}^{1}(D(S))<A_{z}^{2}(S)+B_{z}^{2}(D(S))$. Given that $B_{z}^{1}(D(S))=$ $B_{z}^{2}(D(S))$, we have that $A_{z}^{1}(S)<A_{z}^{2}(S)$. On the other hand, given that $A_{y}^{1}(S)=A_{y}^{2}(S)$ and $z$ is to the south-east of $y$, we have that $A_{z}^{1}(S)>A_{z}^{2}(S)$, a contradiction.

Lemma 2 says that $K S$ is "sandwiched" between $E A$ and $D E A$ (see Fig. 2). This implies that $K S$ satisfies area-based fairness, a fact that plays a central role in our characterizations.

\section{References}

[1] T.C.A. Anant, B. Mukherji, K. Basu, Bargaining without convexity: Generalizing the Kalai-Smorodinsky solution, Econom. Lett. 33 (1990) 115-119.

[2] N. Anbarc1, Noncooperative foundations of the area monotonic solution, Quart. J. Econ. 108 (1993) 245-258.

[3] N. Anbarc1, Finite alternating move arbitration schemes and the equal area solution, Theory and Decision 61 (2006) 21-50.

[4] N. Anbarci, J.P. Bigelow, The area monotonic solution to the cooperative bargaining problem, Math. Social Sci. 28 (1994) 133-142.

[5] E. Calvo, H. Peters, Dynamics and axiomatics of the equal area solution, Internat. J. Game Theory 29 (2000) 81-92.

[6] Y. Chun, The equal-loss principle for bargaining problems, Econom. Lett. 26 (1988) 103-106

[7] J.P. Conley, S. Wilkie, The bargaining problem without convexity: extending the egalitarian and Kalai-Smorodinsky solutions, Econom. Lett. 36 (1991) 365-369.

[8] E. Kalai, Proportional solutions to bargaining situations: interpersonal utility comparisons, Econometrica 45 (1977) 1623-1630.

[9] E. Kalai, M. Smorodinsky, Other solutions to Nash's bargaining problem, Econometrica 43 (1975) 513-518.

[10] J.F. Nash, The bargaining problem, Econometrica 18 (1950) 155-162.

[11] S. Rachmilevitch, Efficiency-free characterizations of the Kalai-Smorodinsky bargaining solution, Oper. Res. Lett. 42 (2014) 246-249.

[12] A.E. Roth, Independence of irrelevant alternatives, and solutions to Nash's bargaining problem, J. Econom. Theory 16 (1977) 247-251.

[13] A.E. Roth, An impossibility result concerning n-person bargaining games, Internat. J. Game Theory 8 (1979) 129-132.

[14] Y. Xu, N. Yoshihara, Alternative characterizations of three bargaining solutions for nonconvex problems, Games Econom. Behav. 57 (2006) 86-92. 